\title{
Single-Step Step Ladder Expansive Cranioplasty
}

\author{
S. K. Sengupta ${ }^{1}$ \\ ${ }^{1}$ Command Hospital (EC), Kolkata, West Bengal, India \\ Indian J Neurosurg 2017;6:107-112.
}

\begin{abstract}
Address for correspondence Col. S. K. Sengupta, MS (Gen Surg), MCh (Neurosurgery), 349/2; Dakshin Behala Road, Kolkata, West Bengal 700061, India (e-mail: sudipkumarsengupta14@rediffmail.com).
\end{abstract}

Abstract
Keywords
- subdural hematoma
- single-step step
ladder
- expansive
cranioplasty

Background Recently, a concept of an "expansive cranioplasty" was forwarded as a feasible single-step alternative to performing a decompressive hemicraniectomy followed by cranioplasty at a later date.

Materials and Methods Procedure of single-step step ladder expansive cranioplasty following craniotomy with removal of subdural hematoma (SDH) was done in a case of acute SDH at our center. The author presents the clinical presentation, operative steps, pre- and postoperative neuroimagings, and outcome of the case.

Results Postoperative NCCT of the head film showed an increase in biparietal diameter by $10 \mathrm{~mm}$. Measured from the craniectomy margin, the distance of the inner table of the bone was $2.81 \mathrm{~mm}$. Preoperative midline shift of $10.2 \mathrm{~mm}$ reduced to $7.9 \mathrm{~mm}$, whereas the GCS improved from E1VTM3 to E2VTM5 in the immediate postoperative period. On his review at the end of 3 months, the patient had no surgery-related complication.

Conclusion "Step ladder expansive cranioplasty" has a promise and deserves a trial in more number of cases. If proven acceptable, it can be especially useful for patients for whom coming back for a second surgery may not be all that easy.

\section{Introduction}

Decompressive hemicraniectomy has been frequently used in management of conditions with refractory raised intracranial pressure (ICP). ${ }^{1-3}$ A concept of a "step ladder expansive cranioplasty" was proposed recently ${ }^{4,5}$ to achieve its objective of reducing ICP and at the same time avoiding a second surgery in the form of a cranioplasty and the complications associated with it. ${ }^{6}$ Being able to put the bone flap back in the same sitting as the craniotomy would also ensure avoidance of trephination syndrome, a wellknown accomplice of decompressive hemicraniectomy. ${ }^{7}$ The author performed a single-step step ladder cranioplasty following craniotomy and evacuation of acute SDH with a satisfactory outcome. The clinical presentation, operative steps, pre- and postoperative neuroimagings, and outcome of the case are presented here.

\section{Materials and Methods}

A 72-year-old man, a known case of hypertension, chronic obstructive pulmonary disease (COPD), and type 2 diabetes mellitus, was brought to the emergency department with a history of an accidental fall in the bathroom. On arrival, the patient was recorded to have a Glasgow coma scale (GSC) score of E2V2M5. There were no lateralizing signs. NCCT of the head revealed an acute right-sided frontoparietotemporal SDH with a midline shift of $10.2 \mathrm{~mm}$ to the left (-Fig. 1A).

During his workup for surgery, the patient deteriorated further to a GCS score of E1VTM3. However, even at this stage, there were no lateralizing sign.

A written informed consent was obtained from the next of kin and the patient was taken up for an emergency craniotomy with evacuation of SDH followed by a singlestep step ladder expansive cranioplasty in the same sitting.

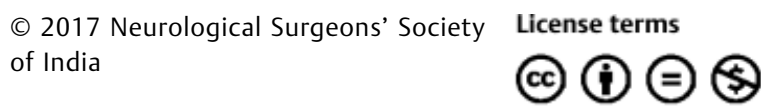

received

December 23, 2016 accepted after revision

February 7, 2017

published online

May 19, 2017
DOI https://doi.org/

10.1055/s-0037-1602755. ISSN 2277-954X. 


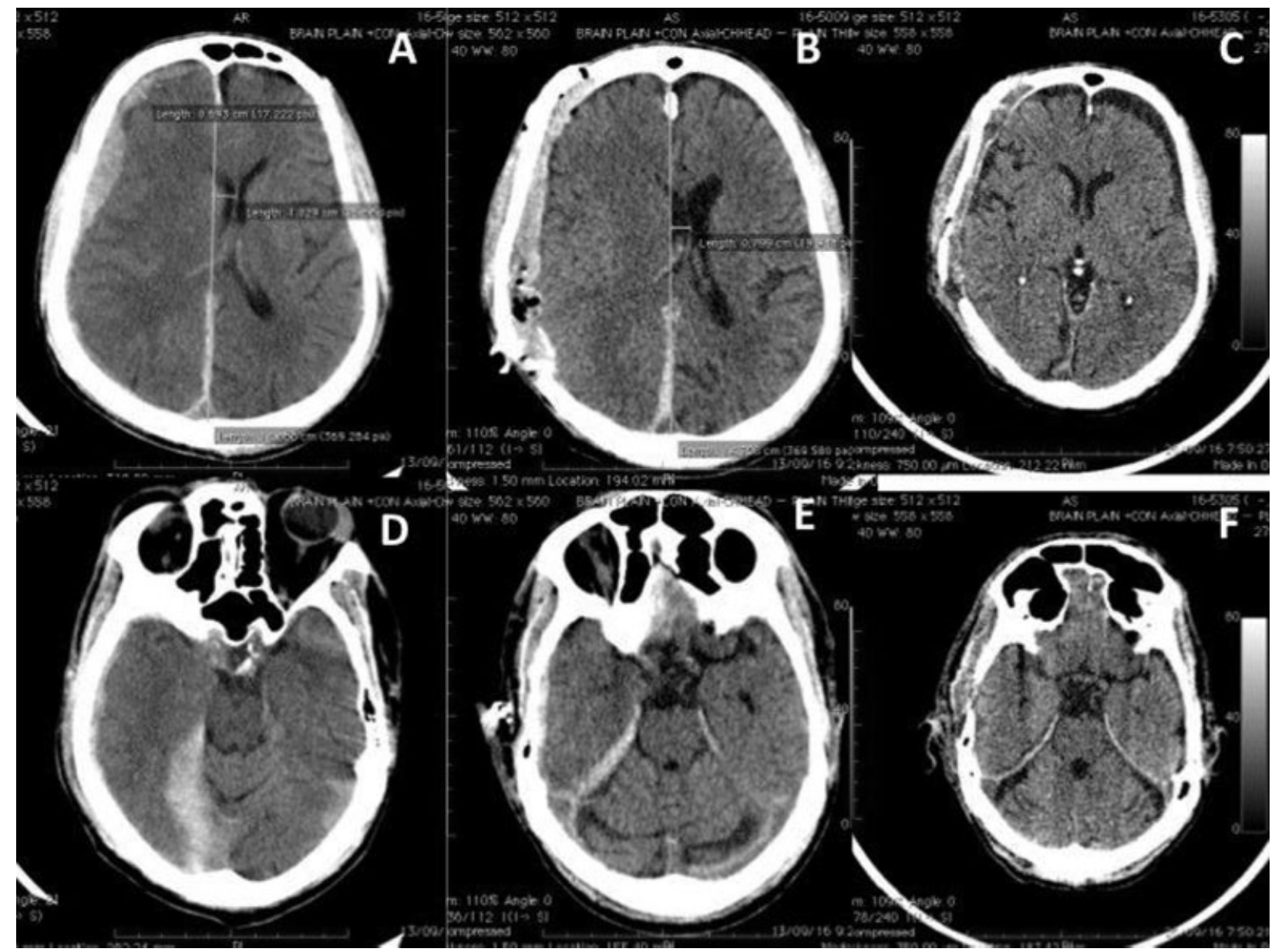

Fig. 1 Axial sections of NCCT of the head. (A) Preoperative image showing right frontoparietotemporal acute SDH with 10.2-mm midline shift. (B) Midline shift of $0.8 \mathrm{~mm}$ on first postoperative day. (C) Complete resolution midline shift evidenced on third postoperative week. (D-F) Axial section of NCCT of the head at the level of the basal cisterns preoperatively, on first postoperative day and on third postoperative week, respectively.

\section{Operative Steps}

In supine position with the head turned to contralateral side, a standard right-sided question mark incision was made extending anteriorly to the hairline. The anterior end of this line was connected to the most posterior point approachable on the contralateral superior temporal line by a curvilinear incision with convexity to the left side, completing an inverse S-shaped scalp incision (-Fig. 2A). While a standard trauma flap was raised and turned laterally, a second subgaleal flap was raised using the contralateral extension of the incision, to be used as a rotation flap subsequently (-Fig. 2B). A $12-\times 08-\mathrm{cm}$ frontotemporoparietal (FTP) free bone flap was raised and remaining part of the squamous part of the temporal bone nibbled off.

After a cruciate durotomy and evacuation of SDH, a bleeding cortical vessel was cauterized. After securing hemostasis, duraplasty was done with a pericranial patch. Bone flap for expansive cranioplasty was prepared by (1) smoothening off the bony edges and (2) fixing titanium miniplates on the inner table (-Fig. $2 \mathbf{C}$ ).
The construct was placed back over the craniectomy defect as a cap and fixed with miniplates and screws on the outer table (-Fig. 3C,D), creating a single-step step ladder cranioplasty. Temporalis muscle with fascia was sutured to a thin rim of the temporalis muscle and fascia, left over the superior temporal line (-Fig. 2D). The medial scalp flap was rotated laterally and sutured to the lateral scalp flap covering the cranioplasty construct. A wide subgaleal undermining was done and remaining wound on the medial side was closed in two layers (-Fig. 2E) over a suction drain.

Postoperative follow-up: The patient was continued with elective ventilation postoperatively along with cerebral decongestive measures. He was shifted to intensive care unit (ICU) and put on a continuous monitoring of the vital signs and a two hourly neurologic examinations, with the neurosurgical team and operating theater prepared for removal of the bone flap to convert the surgery into a formal decompressive hemicraniectomy in case of any clinical deterioration.

After first 48 postsurgery hours, because the patient showed neurologic improvement, frequency of neurologic 


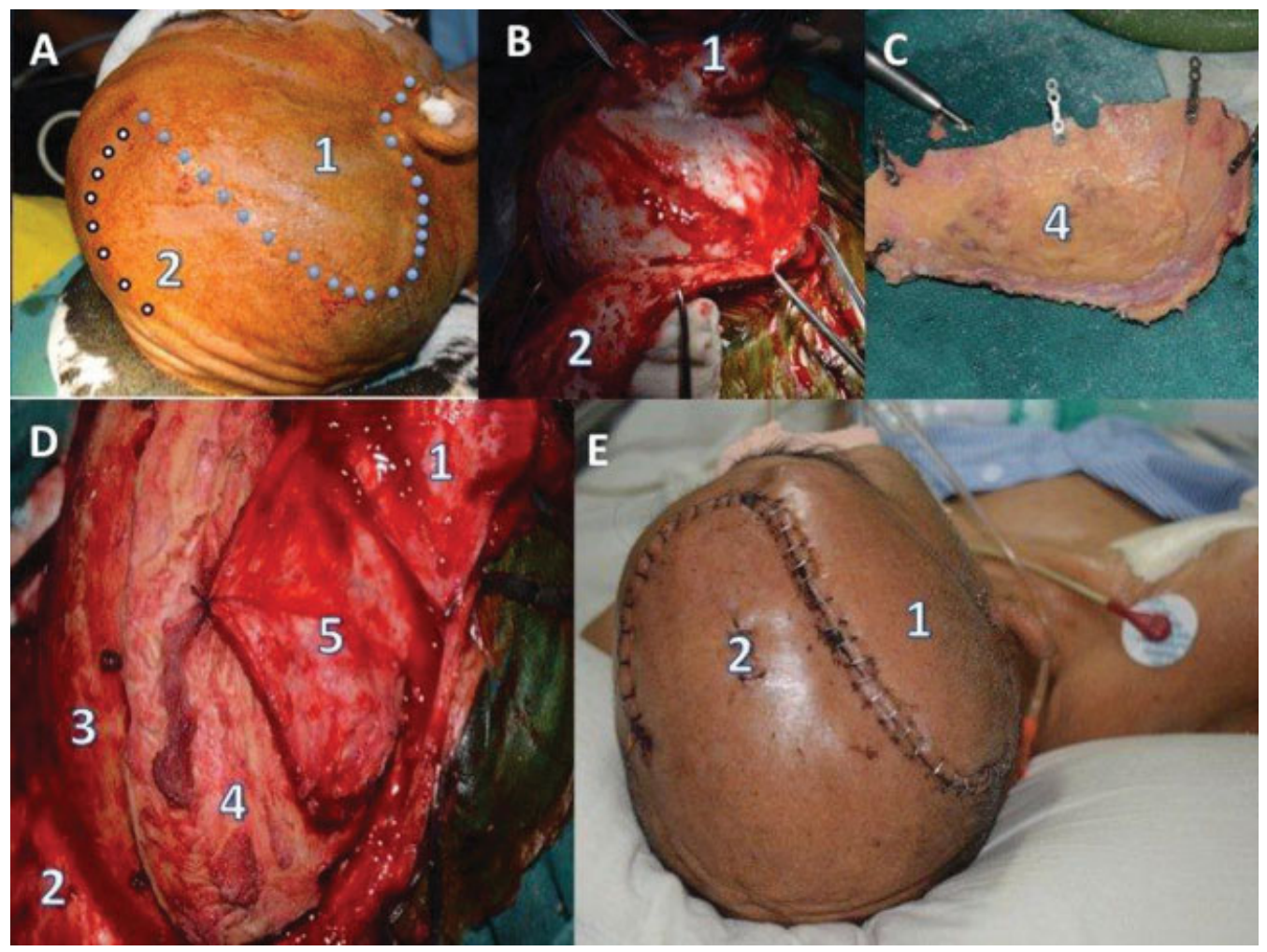

Fig. 2 Clinical photographs: (A, B) Operative and (E) postoperative photographs of the scalp flaps. Blue dots indicate the standard question mark incision line with an inverted S-shaped extension along the white dots to cater for a rotation flap. (1) The standard subgaleal scalp flap raised. (2) The medial subgaleal flap used during closure as a rotation flap. (C) Medial view of the free craniectomy bone flap with the titanium miniplates fixed to its medial surface with screws. (D) Perioperative photograph showing the single-step step ladder expansive cranioplasty construct covering the craniectomy defect (1) and (2) scalp flaps as defined above. (3) Cranium at the margin of the craniotomy defect and (4) craniectomy bone flap. (5) Temporalis muscle with temporalis fascia covering the temporal craniectomy defect.

monitoring was reduced to four times a day. NCCT of the head was done on the immediate postoperative period and repeated on the third week postsurgery.

\section{Results}

The patient remained hemodynamically stable and showed substantial neurologic recovery with a GCS of E2M5VT recorded in the immediate postoperative period, which further improved to E4M6VT by third postoperative day. However, he could not withstand trials for extubation and required tracheostomy in view of prolonged ventilation on fifth postoperative day. He developed hospital-acquired pneumonia with Klebsiella pneumoniae grown in tracheal aspirate and developed features of sepsis. With parenteral antibiotics and supportive care, he improved gradually and ventilatory support could be taken off 6 weeks postsurgery. Over the next 2 weeks, tracheostomy decannulation was done and the patient could be discharged home, ambulant without support and on normal diet.

Immediate postoperative NCCT of the head ( $\mathbf{F i g} . \mathbf{1 B}, \mathbf{E}$ ) revealed an extradural collection while there was reduction in the extent of the midline shift $(8 \mathrm{~mm})$ and resolution of uncal herniation. Both the extradural collection and midline shift resolved in 2 weeks' time, as evidenced by the NCCT of the head obtained on the third postoperative week
( - Fig. 1C, F). Measured from the midline in the axial NCCT images in the bone window ( - Fig. 2A), the distance of the parietal eminence was found to have increased by $10.2 \mathrm{~mm}(72.8 \mathrm{~mm}$ on the effected side as compared with $62.6 \mathrm{~mm}$ on the normal side) as a result of the expansive cranioplasty. Measured from the craniectomy margin, lateral limit of the dural outpouching was $28.1 \mathrm{~mm}$ (-Fig. 3A). While there was neurologic recovery, the patient continued to require prolonged ventilatory support in view of his chest infection .

At the time of his review at 3 months, the patient did not have any persistent headache, vomiting, or cerebrospinal fluid (CSF) rhinorrhea. He complained of altered sleep pattern with inability to concentrate and irritability. Caregivers volunteered history of the individual talking irrelevantly and crying at times and difficulty in finding appropriate words. He had an MMSE (Mini-Mental State Examination) of $12 / 30$, and there was no focal neurologic deficit. There were no operative site-related complications.

\section{Discussion}

Requirement of a second surgery in the form of cranioplasty and various neurologic complications, popularly grouped as "trephination syndrome," have always been a matter of concern, in spite of a proven beneficial role of decompressive 


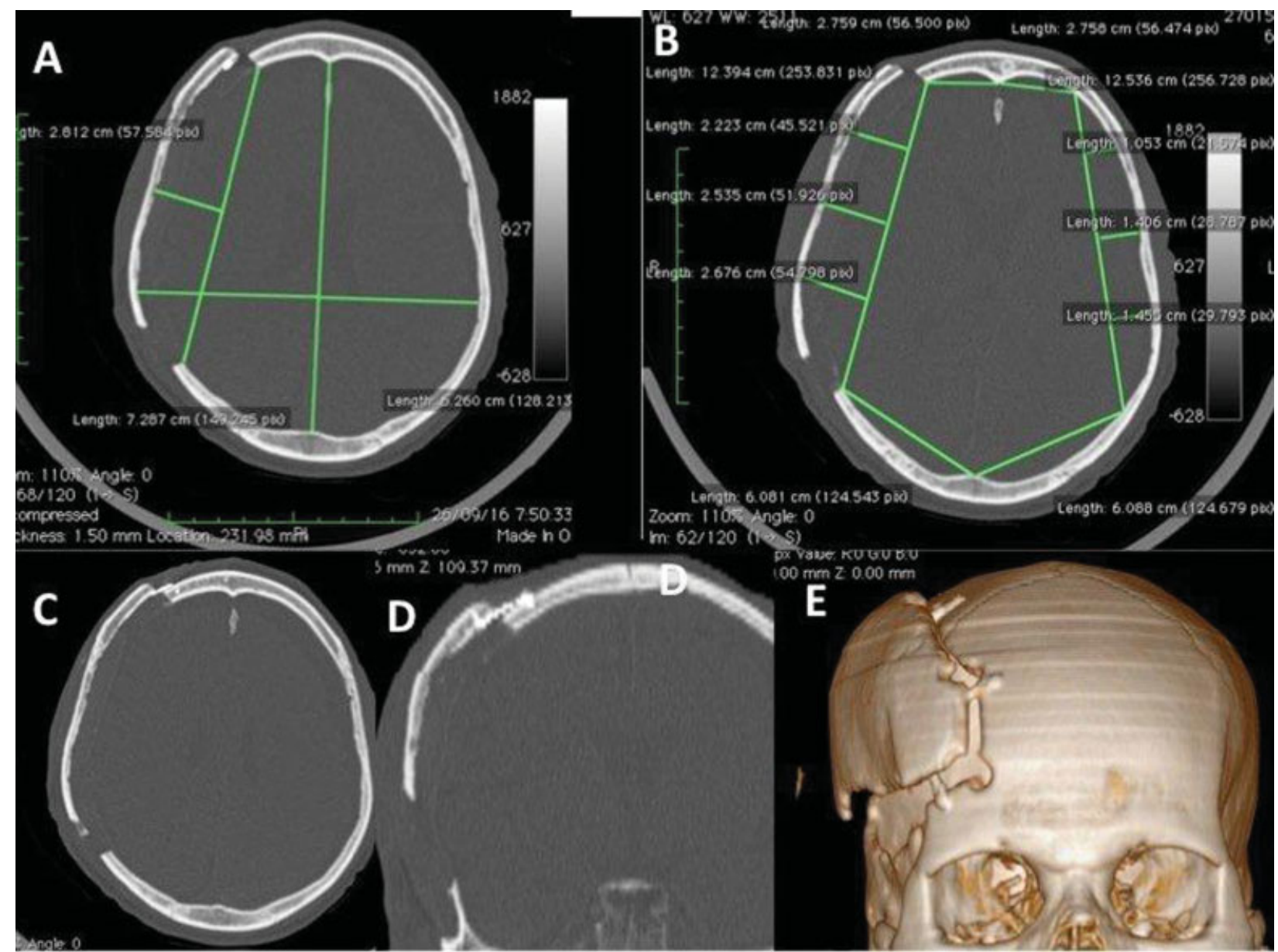

Fig. 3 Step ladder cranioplasty: Postoperative NCCT images. (A) Axial section showing an increase in biparietal diameter of $10 \mathrm{~mm}$ on the cranioplasty side. Outer margin of the brain measured to be $2.81 \mathrm{~cm}$ beyond the level of the craniectomy defect. (B) Measured from the craniectomy edges, the average distance of the inner table has increased by $12 \mathrm{~mm}$ as compared with the contralateral side. (C, D) Axial and coronal sections in bone window showing a titanium miniplate with the cranium around the craniectomy defect and the craniectomy flap fixed on its opposite sides with screws. (E) Three-dimensional volumetric reconstruction images showing the step ladder cranioplasty construct.

hemicraniectomy in a wide range of pathologic conditions causing refractory raised ICP. Cranioplasty, apart from adding cost and inconvenience of a second surgery, can add to its share of complications.

Efforts have been made to alleviate some of these problems by resorting to various alternative, novel surgical techniques, and modifications. ${ }^{8-10}$

However, they have been conducted in very small number of cases, and none have measured the ICP reduction or volume expansion achieved. The concern that leaving the bone flaps back under the scalp flap will restrict the available space and put additional pressure on the brain parenchyma has not been addressed in any of these studies. This has resulted in lack of acceptance of these techniques in general practice.

Recently a concept of an expansive cranioplasty was proposed as a credible alternative, based on a mathematical model. ${ }^{4}$ The aim of this study was to find out the volume expansion, and hence projection of dural outpouching beyond the craniectomy margin was required to be achieved to translate into an adequate ICP reduction and then to work out a method to allow adequate intracranial volume expansion while at the same time being able to put the bone flap back. In this study the author searched available literature and found three studies that compared the craniectomy size with the volume expansion achieved. ${ }^{11-13}$ A cumulative number of 104 cases of unilateral decompressive hemicraniectomy were reported in the three studies. Because none of the studies had recorded the preoperative location of the dura/inner table of the skull and all the measurements were taken from the craniectomy margin, the author devised a mathematical model that could represent cranial cavity. Based on this mathematical model that hypothesized the intracranial space, to be geometrically equivalent to a $8.945-\mathrm{cm}$ radius hemisphere (volume $1,500 \mathrm{~cm}^{3}$ ), it was worked out that in a unilateral FTP decompressive craniectomy for an oval craniectomy defect of $12 \times 8 \mathrm{~cm}$ (equivalent to a circular defect of $9.79 \mathrm{~cm}$ diameter) has a surface area of $75.42 \mathrm{~cm}^{2}$ and the maximum distance from the line joining the craniectomy margins to the outer margin of unexpanded dura is $1.45 \mathrm{~cm}$. The craniectomy defect in our patient was 
$12 \times 8 \mathrm{~cm}$ in size. When an imaginary line was drawn on the contralateral side at the level of the craniectomy, the maximum distance of the inner table from the line was $1.45 \mathrm{~cm}$ (-Fig. 3B), which validates the assumptions of the mathematical model.

The volume of dural outpouching in this patient, when measured from the craniectomy margin $(2 / 3 \times$ surface area of the defect $\times$ height of the dural outpouching from the craniectomy defect), was $141.28 \mathrm{~cm}^{3}$, because the height of the dural outpouching from the craniectomy margin was found to be $2.81 \mathrm{~cm}$ (-Fig. 3A). The maximum volume expansions achieved by a unilateral decompressive hemicraniectomy recorded by Cavuşoğlu $\mathrm{H}$ et al and Olivecrona $\mathrm{M}$ et al were $102.7 \mathrm{~cm}^{3}$ and $109 \mathrm{~cm}^{3}$, respectively. ${ }^{11,13}$ Though the study by Münch E et al recorded $157.6 \mathrm{~cm}^{3}$ as the maximum volume expansion achieved, the mean volume expansion reported was $92.6 \mathrm{~cm}^{3}$.

In an anatomical study based on NCCT films by Sengupta et $a{ }^{5}{ }^{5}$ it was noted that, after decompressive hemicraniectomy, the increase in the width of the dural outpouching, from the preoperative state (presumed in the study to be represented by the dura on the unoperated side), varied from 0.7 to $1.4 \mathrm{~cm}$, which is inversely proportional to the size of the craniectomy defect $(0.7 \mathrm{~cm}$ for an oval craniectomy defect of $10.8 \times 8.5 \mathrm{~cm}$ with a surface area of 72.12 ). Based on the method used by the author in calculating the increase in width of the dural outpouching as compared with the contralateral side ( $\mathbf{F}$ Fig. $\mathbf{3 B}$ ), in this patient, there was a $1.15-\mathrm{cm}$ increase in the width.

In a recent publication, in the 26 patients who underwent decompressive hemicraniectomy for acute SDH, a mean postoperative midline shift of $9.1 \mathrm{~mm}$ was noted in the postoperative NCCT of the head images obtained on the third postoperative day. ${ }^{14}$ Our patient was recorded to have a midline shift of $8.0 \mathrm{~mm}$ on the first postoperative day, which was found to have resolved completely in the neuroimaging obtained on the third postoperative week. In the same study, swelling above bone flap margin was obtained by drawing an imaginary line drawn congruent with measurement of contralateral hemisphere to represent the absent bone flap and brain tissue above the imaginary line was measured. Mean measurement of swelling above the preoperative limit of the bone flap in this study was recorded to be $9.6 \mathrm{~mm}$. In our case the lateral displacement of the calvarial inner cortex as a result of the expansive cranioplasty was recorded to be $1.1 \mathrm{~mm}$. When measured from the craniectomy margin, lateral margin of the brain was recorded to be 2.81 $\mathrm{cm}$, as compared with 2.85 to $3.8 \mathrm{~cm}$ reported by Cavuşoğlu et al. ${ }^{11}$

In absence of concurrent ICP measurement, though the intracranial volume augmentation is obvious, neurologic recovery cannot be ascribed solely to the "expansive cranioplasty." Expansive cranioplasty increases the surface area of the skull, while causing intracranial volume augmentation and requiring the scalp to cover a wider surface area as compared with the preoperative state. Rotation scalp flap devised in this case took care of the increased cranial surface area successfully.

\section{Concerns}

- Bony nonunion and possibility of implant failure: In the limited follow-up of 3 months, there is no radiologic evidence of bony nonunion. At the same time, there is no evidence of implant failure either. Theoretically, the hydrostatic force of the CSF-containing dural bag, counteracting any inward force acting on the cranioplasty construct, lend some support and reduces the likelihood of implant failure.

- Cosmesis: A bony elevation and resulting asymmetry of the skull will be an unavoidable accomplice. However, given a choice, the patient may prefer to have a wellcontoured bony elevation as compared with a sunken scalp flap as a deformity. Even if a redo surgery is required for cosmetic reason, a step ladder expansive cranioplasty will have following advantages:

$>$ Avoidance of complications of trephination syndrome $>$ Avoiding surgery at recipient site for bone flap preservation

$>$ Protecting the brain at the craniectomy site until the patient is taken up for revision cranioplasty

- Propensity to develop chronic SDH: Relative increase in the cranial volume as compared with the brain volume leads to CSF hygroma and is likely to increase the propensity to develop chronic SDH.

- Possibility of bilateral surgery: In view of the increase in cranial surface area, a bilateral expansive cranioplasty will require extensive reconstructive measures to achieve scalp cover, way beyond the scope of an emergency surgery.

Issue of superiority over existing procedure requires to be addressed by operating upon and following up more number of cases for a longer time.

Caution: Not to be performed in brain contusion and lobar ICH in which the volume expansion required to achieve satisfactory reduction in ICP is yet to be worked out.

\section{Conclusion}

"Step ladder expansive cranioplasty" has a promise to provide a credible single-step alternative to decompressive hemicraniectomy followed by cranioplasty at a later date. It deserves a trial in more number of cases. If proven acceptable, it can be especially useful for patients from remote places with limited access to neurosurgical services.

\section{References}

1 Jiang JY, Xu W, Li WP, et al. Efficacy of standard trauma craniectomy for refractory intracranial hypertension with severe traumatic brain injury: a multicenter, prospective, randomized controlled study. J Neurotrauma 2005;22(06):623-628

2 Bao YH, Liang YM, Gao GY, Pan YH, Luo QZ, Jiang JY. Bilateral decompressive craniectomy for patients with malignant diffuse brain swelling after severe traumatic brain injury: a 37-case study. J Neurotrauma 2010;27(02):341-347 
3 Yoo DS, Kim DS, Cho KS, Huh PW, Park CK, Kang JK. Ventricular pressure monitoring during bilateral decompression with dural expansion. J Neurosurg 1999;91(06):953-959

4 Sengupta SK. Step-ladder expansive cranioplasty after decompressive craniotomy and expansive duraplasty: discussing possibilities on a mathematical model. Indian J Neurosurg 2015;4:15-21

5 Sengupta SK, Sahni H, Rappai TJ, Verma S. Step ladder expansive cranioplasty: feasibility study on computed tomographic scan images. Indian J Neurosurg 2015;4:102-108

6 Gooch MR, Gin GE, Kenning TJ, German JW. Complications of cranioplasty following decompressive craniectomy: analysis of 62 cases. Neurosurg Focus 2009;26(06):E9

7 Kurland DB, Khaladj-Ghom A, Stokum JA, et al. Complications associated with decompressive craniectomy: a systematic review. Neurocrit Care 2015;23(02):292-304

8 Schmidt JH III, Reyes BJ, Fischer R, Flaherty SK. Use of hinge craniotomy for cerebral decompression. Technical note. J Neurosurg 2007;107(03):678-682

9 Valença MM, Martins C, da Silva JC, et al. In: Balestrino M, ed. An Innovative Technique of Decompressive Craniectomy for Acute
Ischemic Stroke: Advances in the Treatment of Ischemic Stroke; 2012

10 Peethambaran AK, Valsalmony J. Four-quadrant osteoplastic decompressive craniotomy: a novel technique for decompressive craniectomy avoiding revision cranioplasty after surgery. Neurol India 2012;60(06):672-674

11 Cavuşoğlu H, Kaya RA, Türkmenoğlu ON, Aydin Y. Value of early unilateral decompressive craniectomy in patients with severe traumatic brain injury. Ulus Travma Acil Cerrahi Derg 2010;16 (02):119-124

12 Münch E, Horn P, Schürer L, Piepgras A, Paul T, Schmiedek P. Management of severe traumatic brain injury by decompressive craniectomy. Neurosurgery 2000;47(02):315-322, discussion $322-323$

13 Olivecrona M, Rodling-Wahlström M, Naredi S, Koskinen LO. Effective ICP reduction by decompressive craniectomy in patients with severe traumatic brain injury treated by an ICPtargeted therapy. J Neurotrauma 2007;24(06):927-935

14 Kwon YS, Yang KH, Lee YH. Craniotomy or decompressive craniectomy for acute subdural hematomas: surgical selection and clinical outcome. Korean J Neurotrauma 2016;12(01):22-27

Erratum: This article has been updated as per the Erratum; doi: 10.1055/s-0037-1606263. The article title has been changed to "Single-Step Step Ladder Expansive Cranioplasty". 